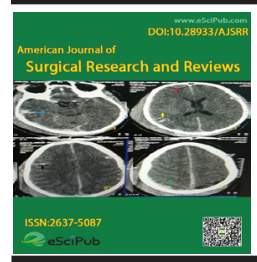

American Journal of Surgical Research and Reviews (ISSN:2637-5087)

\title{
Mini-thoracotomy versus Full Sternotomy Techniques in Mitral Valve Surgery: Blood Loss, Wound infections, Post-operative Recovery, Morbidity and Mortality Investigation
}

Issaka Zalle ${ }^{1 *}$, Moussa Son ${ }^{1}$, Mouhcine El Mardouli ${ }^{1}$, Mohamed EL-Alaoui ${ }^{1}$, Macedoine Nijimbere ${ }^{1}$, Abdoulaziz Thiombiano", Drissi Boumzebra ${ }^{1}$

${ }^{1}$ Cardiovascular Surgery, Mohammed VI University Hospital, Marrakech, Morocco.

\section{ABSTRACT}

Background: Mitral valve surgery is routinely performed through a Median full sternotomy (MFS) with excellent long term outcomes. Minimally invasive mitral (MIMVS) valve surgery is also a surgical approach that improves operative outcomes. In this study we report early post-operative outcomes in minimally invasive mitral valve surgery compared with MFS access with reference to Blood Loss, Wound infections, post-operative Recovery, Morbidity, Mortality and others variables. Patient and Methods: This study was a prospective data collection from 52 consecutive patients who underwent isolated mitral valve surgery at our institution from November 2017 to October 2019. Population study was divided to two groups, MIMVS (group I $n=26$ ) and MFS (group II $n=26$ ). Pre-operative planning were performed so that to obtain similar characteristics. Intra and post-operative data were analysed. Results: The baselines characteristics were similar in both groups. Of the 26 patients in group I, 23 (88.46\%) underwent mitral valve replacement and 3 a mitral valve repair. All the patients in group II underwent mitral valve replacement. There was no difference in term of mortality and morbidity. MIMVS was associated with longer CPB time (mean 161.9 vs 89.8 mins, $P=.025$ ) but similar ACC (99 mins vs $64 \mathrm{P}=.468$ ) time. MIMVS Patients had likely lower incidence of red blood cells transfusion (12.2\% vs $34.7 \%$,), post-operative haemoglobin was similar before transfusion. Haemorrhage complications were more likely in the group II (26.08 vs $7.7 \%$ ); requiring inotropic support was found to be higher in the group

*Correspondence to Author: Issaka Zalle

Cardiovascular Surgery, Mohammed VI University Hospital, Marrakech, Morocco.

How to cite this article:

Issaka Zalle, Moussa Son, Mouhcine El Mardouli, Mohamed ELAlaoui, Macedoine Nijimbere, Abdoulaziz Thiombiano, Drissi Boumzebra. Minithoracotomy versus Full Sternotomy Techniques in Mitral Valve Surgery: Blood Loss, Wound infections, Post-operative Recovery, Morbidity and Mortality Investigation. American Journal of Surgical Research and Reviews, $2021 ; 4: 21$.

\section{eScîPub}

eSciPub LLC, Houston, TX USA. Website: https://escipub.com/

By using the site/services, you are agreeing to our Policies:

https://escipub.com/terms-privacypolicy-disclaimer/ 
II (54.5 vs 19\%). In addition, patients in the MIMVS group had a shorter mechanical ventilation time (1.6 [1-6] vs 3.6 [2-8] hours; $P<.01$ ), shorter ICU stay 1.36 [1-6] vs 3.6 [2-8] days, $p<.01$. Length of hospital stay and chest tube stay were found to be shorter, respectively 6.9 [6-16] vs 7, 7 [7-13] and (1.38 [1-2] days vs 2.64 [2-4], $\mathrm{P}<.01)$. Wound infections were not found in both groups. Conclusion: Although the controversy interest of minimally invasive mitral valve surgery, it may be associated with less blood loss, faster post-operative recovery but increases operation time.

Keywords: Full sternotomy; Right anterior minithoracotomy; Mitral valve surgery; Early outcomes

\section{INTRODUCTION}

The prevalence of heart valve disease has been increasing in many developed countries. Surgical approach may be the main treatment for advanced heart valve disease [1]. Traditionally; heart valve surgery has been performed through a conventional sternotomy with cardio- pulmonary bypass (CPB). This traditional approach allows easy access of the mitral valve and so allows surgeons to avoid certain complications.

Since its introduction in mid-1990 [2-3], minimally invasive valve surgery (MIVS) has become a surgical option with the development of techniques and technologies during the past decades. The advantage in minimally invasive mitral valve surgery (MIMVS) remains topical, with encouraging results ${ }^{[4-5]}$. Certain retrospective studies revealed that the potential benefits of MIMVS can be obtained uneventful without increased morbidity or mortality [6-7]. According to the literature ${ }^{[8]}$, it may lead to inferior results particularly for mitral valve surgery (MVS).

The aim of this study was to report the early post-operative outcomes in MIMVS compared to the access by full sternotomy in our department of cardiovascular surgery.

\section{PATIENTS AND METHODS}

Among the patients admitted to our Department for heart valve surgery, a total of 52 patients planned for isolated mitral valve surgery were selected to perform this study. The patient's selection for MIMVS was used. A prospectively collected data was analysed from November 2017 to October 2019. The patients were divided to two groups, the group I $(n=26)$ underwent MIMVS and in the group II $(n=26)$ was operated with MFS. Pre-operative planning was performed so that to obtain similar population studies between the two groups. There were no statistical differences between groups in terms of demographic data, cardiac status, and associated pathologies. All peri- operative data and in-hospital outcomes were analysed. Emergency surgery cases and Patients with concomitant procedures were excluded from this study. The clinical and paraclinical data of the two groups were collected including 30-day post-operative. Our assessment was based on Blood Loss, Wound infections, Early Recovery Morbidity and Mortality.

\section{SURGICAL TECHNIQUE}

Conventional general anesthesia was used in all patients according to the surgical approach and monitoring lines are placed.

In the group I, endotracheal tube is exchanged for a double-lumen tube to allow selective ventilation. Cardiopulmonary bypass (CPB) was instituted via femoral arterial and venous cannulation through 3-4 cm transverse incisions in the right groin. The venous cannula was positioned under trans-oesophageal echocardiographic (TOE) guidance in the right atrium. The majority of cases used mild hypothermia to normothermia CPB. A right lateral mini-thoracotomy, $5-6 \mathrm{~cm}$ incision is made in the anterior axillary line of the 4th intercostal space just superior to the submammary crease. The right lung is deflated to access the pleural cavity, and then soft tissue retractor is placed in the wound. CPB was used throughout the procedure. A video camera was inserted through a $10 \mathrm{~mm}$ port in the right 2 nd intercostal space and a 
transthoracic Chitwood aortic cross-clamp was inserted through a $5 \mathrm{~mm}$ incision in the 3rd intercostal space ${ }^{[5]}$. Antegrade cold blood cardioplegia was administered directly into the aortic root, and then repeated in 20-25 min. The operation field was flooded with carbon dioxide through the camera port. The left atrium was opened posterior to the interatrial groove. The mitral valve was exposed using a left atrial retractor. Minimally invasive tools were utilized. Standard mitral valve surgery techniques were employed. Mitral valve repair procedures were performed following Carpentier techniques ${ }^{[9-10]}$. A temporary right ventricular pacing wire was placed before removing aortic cross clamp.

For the group II patients a MFS was performed and they underwent aortobicaval cannulation for standard CPB. After aortic cross clamping, cold blood cardioplegia infusion was administrated in antegrade fashion into the aortic root. The left atrium was opened posterior to the interatrial groove. The mitral valve was exposed using left atrial retractor. Mild hypothermia to normothermia was the core temperature. Mitral valve replacement procedures were performed by traditional fashion. A temporary right ventricular pacing wire was placed.

\section{RESULTS}

Pre-operative data (table 1).

Pre-operative data showed a mean of age 48.6 [26-66] years in the MIMVS group and 51.4 [20-66] in the FS group, $P$ value was 0.315 . Sex ratio .44 (group I vs 0.22 group II); the mean weight was 56.8[46-76] in the group I, 61.14 [40-84] kg in the group II, P=.393. Left ventricular ejection fraction (LVEF) was $60.55 \%$ [44.5-72] in the group I vs $58.9 \%$ [33-75], $\mathrm{P}=.496$. Mean NYHA was 2.39 [1-3] in the group I vs 2.91 [1-4], $P=.694$.

Table 1 Showing pre-operative data

\begin{tabular}{cccc}
\hline VARIABLES & MIMVS & MFS & P value \\
\hline Number & 26 & 26 & .315 \\
Age, mean and range & $48.6[26-66]$ & $51.4[20-66]$ & 0.44 \\
Sex Ratio & 0.44 & 0.22 & .393 \\
Weight (kg) & $56.8[46-76]$ & $61.14[40-84]$ & 9.1 \\
Artery Hypertension (\%) & 0 & 4.5 & .694 \\
Mellitus diabetes (\%) & 0 & 9.1 & \\
History of smoking & 0 & 0 & \\
Dyslipidaemia (\%) & 0 & $2.91[1-4]$ & \\
NYHA, mean and rang & $2.39[1-3]$ & 52.5 & \\
Mitral stenosis (\%) & 61.53 & 17 & \\
Mitral regurgitation (\%) & 15.38 & 30.5 & \\
Mitral stenosis + mitral regurgitation (\%) & 23.07 & 47 [34-69] \\
Pulmonary Hypertension, mean and \\
range (mmH)
\end{tabular}

In comparison both of the two groups had likely gender. There was also no significant difference similar characteristics about age, weight and in term of heart risks factors and New York 
Heart Association functional class. The mean pre-operative haemoglobin was not different in the two groups (13.6 in group I vs $13.4 \mathrm{~g} / \mathrm{dl}$ in group II). Pulmonary hypertension was found in the two groups without significantly difference, 37 [17-58] $\mathrm{mmHg}$ in the group I, vs 47 [34-69] $\mathrm{mmHg}$ in the group II, $\mathrm{P}=.489$.

Intra and Post-operative data (table 2).
Of the 52 patients, $23(94.23 \%)$ underwent isolated mitral valve surgery. Mitral valve repair (3 patients) was performed in the group I. Patients in the group I had longer cardiopulmonary bypass (mean 161.9 vs 89.8 mins, $P=.025$ ) but similar cross-clamping (99 mins vs $64 P=.468$ ) time.

Table 2 showing Intra and Post-operative data

\begin{tabular}{|c|c|c|c|}
\hline VARIABLES & MIMVS & MFS & $P$ value \\
\hline CPB time, mean and range (mins) & $161.9[126-197]$ & $\begin{array}{c}89.8 \\
{[64-211]}\end{array}$ & .0250 \\
\hline ACC time, mean and range (mins) & 99 [66-162] & $64[41-83]$ & .0468 \\
\hline Red blood cells transfusion (\%) & 12.2 & 34.7 & \\
\hline Haemoglobin, mean and range (g/dl) & $10.6[8.1-13.6]$ & $9.2[7.4-11]$ & 0.488 \\
\hline Inotropic using (\%) & 19 & 54.5 & \\
\hline Inotropic duration, mean and range (days) & $1.53[1-3]$ & $1.67[1-6]$ & .3446 \\
\hline Ventilation time, mean and range (hours) & $3.05[1-6]$ & 6.7 [3-24] & .0011 \\
\hline Length of chest drain stay & $1.38[1-2]$ & $2.64[2-4]$ & .0009 \\
\hline Pulmonary complications (\%) & 11.5 & 8.69 & \\
\hline Cerebrovascular events (\%) & 11.5 & 0 & \\
\hline Haemorrhage complications (\%) & 7.7 & 26.08 & \\
\hline ICU stay, mean and range (days) & $1.36[1-6]$ & $3.6[2-8]$ & $<.0001$ \\
\hline LVEF, mean and range (\%) & $55.25[48-68]$ & $\begin{array}{c}52.35 \\
{[35-65]}\end{array}$ & .4247 \\
\hline Mean aortic gradient mean and range $(\mathrm{mmHg})$ & $4.19[3-8]$ & $\begin{array}{c}3.75 \\
{[2.77-6.6]}\end{array}$ & .3264 \\
\hline Normal function of prosthesis (\%) & 100 & 100 & \\
\hline Mechanical prosthesis (\%) & 88.5 & 100 & \\
\hline Mitral valve repair (\%) & 11.5 & 0 & \\
\hline Bioprosthesis (\%) & 0 & 0 & \\
\hline Reconversion to full sternotomy (n) & 0 & - & \\
\hline In hospital mortality (\%) & $3.84 \%$ & $0 \%$ & \\
\hline
\end{tabular}

There were no patients who required in- MIMVS may be associated with a lower incitra-operative conversion to MFS in the group I dence of red blood cells transfusions $(12.2 \% \mathrm{vs}$ and no re-exploration for bleeding in the both groups.

$34.7 \%$,).

In addition, patients in group I had a shorter mechanical ventilation time (median, 1.6[1-6] 
Vs 3.6[2-8] hours; $P<.01)$. The length of chest tube stay was shorter in the group I (1.38 [1-2] days vs 2.64 [2-4], $\mathrm{P}<.01$ ), length of hospital stay was 6.9 [6-16] days in group I vs $7,7[7-13]$ in group II, $P=.002$, time of ICU stay $1.36[1-6]$ vs $3.6[2-8]$ days, $p<.01$. In the group I, 1 patient underwent chest drainage for pleural effusion. There were no wound infection in the two groups but cerebrovascular event such as agitation was reported in the group I, (11.5\%). Post-operative TTE data showed similar LVEF 55.25 [48-68] vs 52.35 [35-65], $P=.424$ and $\mathrm{mi}-$ tral mean valve pressure gradient $4.19[3-8]$ vs $3.75[2.77-6.6] \mathrm{mmHg}, \mathrm{P}=0.326$ in the two groups. Biologic report did not showed difference in term of post-operative haemoglobin, 10.6[8.1-13.6] $\mathrm{g} / \mathrm{dl}$ in the group I vs 9.2 [7.4-11], $\mathrm{P}=.488$. Moreover, the incidence of requiring inotropic support was found to be higher in the group II (54.5 vs $19 \%$ ). The overall of inhospital 30-day mortality was $0.01 \%$, it was 1 patient deceased by respiratory insufficiency in the group I.

\section{DISCUSSION}

According to the literature, minimally invasive methods have been developed and improved in the past few decades with thoracoscopic cardiac surgery, technology advancing [2]. Traditional MFS cardiac surgery now provides also good surgical vision and outcomes [11-12].

In our area through many comparative studies between MIMVS and MFS we can say that MIMVS improves early post-operative outcomes. Our experience and many others have found some significant difference in certain variables when we compared the both techniques. In fact in our series we did not found significant difference in the two groups about intra-operative and post-operative complications. In term of blood loss, $12.2 \%$ in group I required red blood cells transfusion vs $34.7 \%, P=.439$, Post-operative haemoglobin was $11.6[8.1,13.6]$ vs 9.2 $[7.4,11] \mathrm{P}=.488$. Dogan et al. ${ }^{[11]}$ in their comparative study have found intra-operative and post-operative blood loss to be equivalent with MIMVS and MFS approaches. However the se- ries of Jian Liu et al. ${ }^{[13]}$ reported a significant different about blood loss. In this study intra-operative transfusion was $23.27 \%$ in MIMVS vs $38.12 \% \mathrm{P}=.0002$ and plasma transfusion was higher in the MFS, $P=0.002$. James $S$ et al. ${ }^{[14]}$ reported 24\%, (intra- operative transfusion) and $26 \%$ (post-operative transfusion) as incidence of red blood cells transfusion in MFS approach. These observations showed a controversy in term of haemorrhage complications between both techniques. Whether the technique used to perform mitral valve surgery, intraoperative complications should depend to surgeon team skills. On basic of these observations and our observation minimally invasive approach should be associated with lower haemorrhage complications but may depend to the level of surgeon' training in this new technique. It is evident that small incisions reduce surgical trauma and therefore avoids bleeding.

In additional, whether the MIMVS or MFS, there were no significant difference in term of morbidity and mortality. Our series and others such as Dogan et al. [11], Jian Liu et al. ${ }^{[13]}$ had reported the same observation. Post-operative morbidity and mortality don't depend to the type of procedure but patients comorbidities and peri-operative management.

In our series the intensive care unit (ICU) stay and ventilation time were significantly shorter in the group I. Respectively we found $1.36[1-6]$ vs $3.6[2-8]$ days, $p<.01 ; 3.05[1-6]$ vs $6.7[3-24]$ hours, $P=.001$. Moreover the length of chest tube stay was shorter in the group I (1.38[1-2] vs $2.64[2-4]$ days, $P=.009$ ). Length of hospital stay was 6.9 [6-16] days in group I vs 7, 7 [7-13] in group II, $\mathrm{P}=.002$. Our study is consistent with Jian Liu et al. ${ }^{[13]}$ who reported a shorter hospital stay $5.64 \pm 3.07$ days vs $11.44 \pm 6.71, P<.001$; shorter ICU stay $44.12 \pm 39.51$ hours vs $61.15 \pm 106.01$ hours, $P=.033$; shorter length of chest tube stay $2.59 \pm 1.75 \quad 4.25 \pm 1.91$ days, $P<.001$. These observations show the evidence of fast post-operative recovery in MIMVS. The shorter ventilation time and the shorter length of chest tube stay allow early ambulation. 
In term of wound infections investigations there were no significant difference between the two groups according to our series and others studies ${ }^{[11-13-14]}$.

Otherwise in our study, patients in the group I had longer CPB (mean 161.9 vs 89.8 mins, $P$ $=.025$ ) but similar cross-clamping (99 mins vs $64 \mathrm{P}=0.468$ ) time. That is almost evident in many studies. Jian Liu et al. ${ }^{[13]}$ reported as CPB time 145, $97 \pm 34.65$ vs $92.24 \pm 25.58$ mins, $\mathrm{P}<.001$; and ACC time $93.89 \pm 25.25 \mathrm{Vs}$ $56.42 \pm 18.09$ mins, $P<.001$. Giovanni Mariscalco et al. ${ }^{[15]}$ in their reviews have found in certain studies such as the one of Glauber et al. ${ }^{[16]}$ and Ruttman et al. ${ }^{[17]}$ that CPB and ACC time were found to be longer in MIMVS compared to MFS. We can say that MIMVS is associated with longer operative time. The small field in minimally invasive approach should reduce surgeon' competences in term of speed.

\section{CONCLUSION}

Although the controversy interest of minimally invasive mitral valve surgery, it may be associated with less blood loss, faster post-operative recovery but increases operation time.

\section{REFERENCES}

[1] Minoru Tabata, MD; Toshihiro Fukui, MD; Shuichiro Takanashi, MD. Do Minimally Invasive

Approaches Improve Outcomes of Heart Valve Surgery? (Circ J 2013; 77: 2232 - 2239).

[2] Lillehei CW, Gott VL, Dewall RA, Varco RL. Surgical correction of pure mitral insufficiency by annuloplasty under direct vision. J Lancet 1957;77(11):446-449.

[3] Cohn LH, Adams DH, Couper GS, Bichell DP, Rosborough DM, Sears SP, et al. Minimally invasive cardiac valve surgery improves patient satisfaction while reducing costs of cardiac valve replacement and repair. Ann Surg 1997; 226: 421 - 426.

[4] Byrne JG, Hsin MK, Adams DH, et al. Minimally invasive direct access heart valve surgery. $\mathrm{J}$ Card Surg 2000; 15:21-34.

[5] Cohn LH, Adams DH, Couper GS, et al. Minimally invasive cardiac valve surgery improves patients satisfaction while reducing costs of cardiac valve replacement or repair. Ann Surg 1997;226:421- 8.
[6] Gammie JS, Bartlett ST, Griffith BP. Small-incision mitral valve repair: safe, durable, and approaching perfection. Ann Surg. 2009; 250:409-15.

[7] Greelish JP, Cohn LH, Leacche M, Mitchell M, Karavas A, Fox J, et al. Minimally invasive mitral valve repair suggests earlier operations for mitral valve disease. J Thorac Cardiovasc Surg. 2003;126:365-73.

[8] Joerg Seeburger, Michael Andrew Borger, Volkmar Falk, Thomas Kuntze, Markus Czesla. Thomas Walther, et al. Minimal invasive mitral valve repair for mitral regurgitation: results of 1339 consecutive patients. European Journal of Cardio-thoracic Surgery 34 (2008) 760-765.

[9] Carpentier A. Cardiac valve surgery - the "French correction." J Thorac Cardiovasc Surg 1983;86:323- 47.

[10] Deloche A, Jebara VA, Relland JYM, et al. Valve repair with Carpentier techniques. The second decade. J Thorac Cardiovasc Surg 1990; 99:990 -1002.

[11] Dogan S, Aybek T, Risteski PS, et al. Minimally invasive port access versus conventional mitral valve surgery: prospective randomized study. Ann Thorac Surg 2005; 79:492-8.

[12] Plass A, Grunenfelder J, Reuthebuch O, et al. New transverse plate fixation system for complicated sternal wound infection after median sternotomy. Ann Thorac Surg 2007; 83:1210-2.

[13] Jian Liu, Bo Chen, Yu-Yuan Zhang, Liang Zheng Fang, Bin Xie, Huan-Lei Huang et al. Mitral valve replacement via minimally invasive totally thoracoscopic surgery versus traditional median sternotomy: a propensity score matched comparative study. Ann Transl Med 2019; 7(14):341

[14] James S, Gammie, MD, Yue Zhao, PhD, Eric D. Peterson, MD, MPH, Sean M. O'Brien, PhD, J. Scott Rankin, MD, et al. Less-Invasive Mitral Valve Operations: Trends and Outcomes From The Society of Thoracic Surgeons Adult Cardiac Surgery Database. (Ann Thorac Surg 2010; 90:1401-10).

[15] Giovanni Mariscalco, MD, PhD, and Francesco Musumeci, MD S. Camillo. The Minithoracotomy Approach: A Safe and Effective Alternative for Heart Valve Surgery Department of Heart and Vessels, Cardiac Surgery Unit, Varese University Hospital, Varese; and Department of Cardiac Surgery and Transplantation, Hospital, Rome, Italy. (Ann Thorac Surg 2014; 97:356 64). 
[16] Glauber M, Miceli A, Gilmanov D, et al. Right anterior minithoracotomy versus conventional aortic valve replacement: a propensity score analysis. J Thorac Cardiovasc Surg 2013; 145: $1222-6$.
[17] Ruttman E, Gilhofer TS, Ulmer H, et al. Propensity scorematched analysis of aortic valve replacement by mini-thoracotomy. J Heart Valve Dis 2010; 19:606-14.

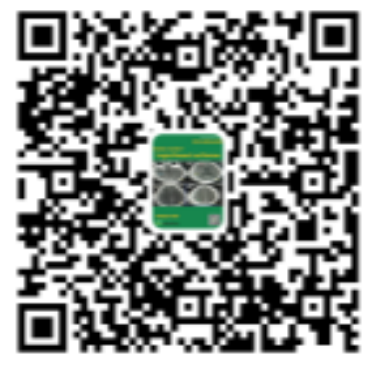

